\title{
IMPLICATIONS FOR THE LATE DIAGNOSIS OF FIBRODYSPLASIA OSSIFICANS PROGRESSIVA: A CASE REPORT
}

Nabila Triântala Bellezzia de Salles ${ }^{1}$,, Isabela Oliveira Vieira da Silva', Paula Consoline Micheletto', Antonio Lucas Lima Rodrigues ${ }^{1}$, Maraísa Centeville', Roberto Marini', Simone Appenzeller ${ }^{1}$

1. Universidade Estadual de Campinas, Campinas (SP), Brazil.

*Corresponding author: nbellezzia@gmail.com

\section{BACKGROUND}

Fibrodysplasia ossificans progressiva (FOP) is a rare genetic disorder which is characterized by widespread heterotopic ossification of connective tissue. The course of the disease is episodic with flare-ups in which patients develop painful localized soft tissue inflammation and tumor-like swellings, in response to numerous possible triggers, such as trauma or illness. The flare-ups and heterotopic ossifications slowly and progressively limit movement and mobility. Almost all FOP cases result from a spontaneous activating mutation in the gene encoding activin receptor IA/activin like kinase-2 (ACVR1/ALK2), a bone morphogenetic protein (BMP). The two classic findings in FOP are hallux valgus and the before-mentioned flare-ups.

\section{CASE REPORT}

Patient, 17 years old, born with bilateral hallux valgus, presented nodulation at the jaw when three months old. At five months old, new lesions appeared in the dorsum, which regressed after circa 20 days. Patient underwent investigation concerning the lesion at the jaw. A biopsy was performed and showed fibrous connective tissue and skeletal muscle tissue, atrophy of focal muscle cells and absence of malignity. A myelogram of two sites was performed and showed no alterations. Thinking of Caffey disease, prednisone $2 \mathrm{mg} / \mathrm{kg} /$ day were prescribed. However, the patient presented no improvement of the lesion and a new bulging lesion appeared in the back. A scintigraphy showed an osteoblastic lesion in the left jaw and soft tissue calcification in the anterior portion of the left thigh. A computerized tomography showed enlargement of the soft tissue in the back. The patient was referred to our service at 11 months old and, noting the hallux valgus and new bone formation lesions, we diagnosed FOP. The family was instructed to avoid trauma and invasive procedures, including intramuscular vaccination as it could be a trigger to a new flare-up. Ibuprofen, naproxen and prednisone were prescribed to treat the flare-ups in different times, but no result was observed. The patient presents an aggressive disease with constant flare-ups and, by the age of 17 , has involvements of the jaw (with significant limitation of oral opening), neck, chest, abdomen, upper and lower limbs. He can only stand on his left leg, but has extreme limitations of movement of the other limbs.

\section{CONCLUSION}

Although FOP is a rare disease, its knowledge is important by all medical professionals, especially pediatricians, orthopedists and general practitioners. Early diagnosis can avoid invasive procedures such as biopsies, that might trigger a flare-up and new bone formation. 